\title{
EFICIÊNCIA DA TERRA DE DIATOMÁCEA NO CONTROLE DE Tribolium castaneum EM MILHO ARMAZENADO
}

\author{
E ffectiveness of diatomaceous earth to control Tribolium castaneum in stored corn
}

\author{
Alberto Luiz Marsaro Júnior ${ }^{a}$, Moisés Mourão Júniorb, Sônia Cristina Pereira Griffelc, \\ Wagner Ribeiro da Silvad, Andréia Esquivel Bressani de Meloe
}

\footnotetext{
a Entomologista, Embrapa Roraima, Boa Vista, RR - Brasil, e-mail: alberto@ cpafrr.embrapa.br

b Bioestatístico, Embrapa Amazônia O riental, Belém, PA - Brasil, e-mail: mmouraojr@ gmail.com

c Acadêmica de Ciências Biológicas das Faculdades Cathedral, BoaVista, RR - Brasil, e-mail: alberto @ cpafrr.embrapa.br

d A cadêmico de Ciências Bioló gicas das Faculdades Cathedral, BoaV ista, RR - Brasil, e-mail: alberto @ cpafrr.embrapa.br

e Acadêmica de Ciências Biológicas das Faculdades Cathedral, BoaVista, RR - Brasil, e-mail: alberto@ cpafrr.embrapa.br
}

\begin{abstract}
Resumo
Tribolium castaneum é uma importante praga do milho armazenado no Brasil. O controle dessa praga é realizado principalmente com inseticidas químicos. $\mathrm{O}$ uso de protetores de grãos na região Norte do Brasil é raro. Nesse sentido, o objetivo desta pesquisa foi avaliar a eficiência da terra de diato mácea aplicada sobre milho armazenado em Roraima para o controle de T. castaneum. G rãos do híbrido BRS 2020 foram tratados com seis dosagens de terra de diatomácea, 0, 200, 400, 600, 800 e 1000 g.t. $t^{-1}$. Cada tratamento, contendo 100 g de grãos, foi infestado com 30 adultos de T. castaneum e mantido em laboratório a $27^{\circ} \mathrm{C}$. Avaliou-se a mortalidade acumulada do $1^{\circ}$ ao $28^{\circ}$ dia. A mortalidade dos adultos foi influenciada pelas dosagens e pelo tempo de exposição dos insetos à terra de diatomácea. Modelos logísticos foram usados para descrever as curvas de mortalidade. Esses modelos foram usados para estimar o tempo necessário para obter 75, 80, 90 e 95\% da mortalidade da população. A dosagem de 1000 g.t $^{-1}$ alcançou 95\% de mortalidade 6 dias após 0 tratamento, a dosagem de 800 g.t. $\mathrm{t}^{-1}$ aos 7 dias, enquanto as dosagens de 600 e 400 g.t ${ }^{-1}$, aos 8 e 10 dias, respectivamente. $\mathrm{Na}$ dosagem de $200 \mathrm{~g}^{-\mathrm{t}^{-1}}$ a máxima mortalidade, obtida após 28 dias de exposição, foi de $42 \%$. As dosagens de 1000 g.t.$^{-1}$ e 800 g.t t $^{-1}$ apresentaram alta eficiência de controle de T. castaneum em um curto espaço de tempo.
\end{abstract}

Palavras-chave: Controle físico. Terra de diatomácea. Milho. 


\begin{abstract}
Tribolium castaneum is important pest of stored corn in Brazil. The control of this pest is realized mainly with chemical insecticides. The use of grain protectants in the north area of Brazil is rare. In this sense, the objective of this research was to evaluate the effectiveness of diatomaceous earth applied in corn stored in Roraima to control T. castaneum. Grains of the hybrid BRS 2020 were treated with six dosages of diatomaceous earth, 0, 200, 400, 600, 800 and 1000 g.t.. Each treatment, containing 100 $g$ of kernels, was infested with 30 adults of $T$. castaneum and maintained in laboratory at $27^{\circ} \mathrm{C}$. Accumulated mortality was evaluated from the $1^{\text {st }}$ to the $28^{\text {th }}$ day. The mortality of adults was influenced by the dosages and the exposure time of insects to diatomaceous earth. Logistic models were used to describe mortality curves. These models were used to estimate the necessary time to obtain 75, 80, 90 and 95\% mortality of the population. The dosage of $1000 \mathrm{~g} \cdot \mathrm{t}^{-1}$ reached 95\% of mortality 6 days after treatment, the dosage 800 g.t t $^{-1}$ at 7 days, while the dosages 600 and 400 g.t. ${ }^{-1}$, at 8 and 10 days, respectively. In the dosage $200 \mathrm{~g} \cdot \mathrm{t}^{-1}$, the maximum mortality resulted after 28 days of exposure, was $42 \%$. Diatomaceous earth in the dosages of $1000 \mathrm{~g} . \mathrm{t}^{-1}$ and $800 \mathrm{~g} \cdot \mathrm{t}^{-1}$ presented high effectiveness to control T. castaneum in a short space of time.
\end{abstract}

Keywords: Physical control. Diatomaceous earth. Corn.

\title{
INTRODUÇÃO
}

T ribolium castaneum (Coleoptera: Tenebrionidae) é umaimportante praga do milho armazenado no Brasil. O controle desse inseto-praga é realizado principalmente por meio do uso de inseticidas químicos. D evido aos problemas de contaminação com resíduos de inseticidas nos alimentos o uso de pós-inertes para o controle de insetos de produtos armazenados tem sido bastante estudado. Existem quatro tipos básicos de pós-inertes: argilas e areias, terra de diatomáceas, sílica aerogel (silicato de sódio) e não derivados da sílica (rochas fosfatadas) (LORINI, 2001).

D entre os pós-inertes, a terra de diatomáceas destaca-se no controle de pragas de grãos armazenados. Diversos estudos já demonstraram o potencial de controle desse produto sobre os principais insetos de armazenamento, Sitophilus granarius (L.) (Coleoptera: Curculionidae) (ALD RY HIM, 1990); O ryzaephilus surinamensis (L.) (Coleoptera: Silvanidae) (ARTHUR, 2001); Plodia interpunctella (Hubner) (Lepidoptera: Pyralidae) (MEWIS; ULRICHS, 2001); R hyzopertha dominica (F.) (Coleoptera: Bostrichidae) (BALD ASSARI; BALD O NI; BARO NIO , 2002); Prostephanus truncatus (Horn) (Coleoptera: Bostrichidae), Callosobruchus maculatus (F.) (Coleoptera: Bruchidae) and Sitophilus zeamais (STATHERS; DENNIFF; GOLOB, 2004); T ribolium castaneum (Herbst) (Coleoptera: Tenebrionidae) (ARNAUD et al., 2005); Sitophilus oryzae(L.) (Coleoptera: Curculionidae) (ATHANASSIO U et al., 2005a); A canthoscelides obtectus (Say) (Coleoptera: Bruchidae) (BELLO et al., 2006) e E phestia kuehniella Zeller (Lepidoptera: Pyralidae) (COLLINS; COOK, 2006).

A terra de diatomácea é obtida de depósitos de carapaças de algas diatomáceas oriundas da era Cenozoica, constituídas predominantemente de sílica amorfa (dióxido de sílica) (SUBRAMANYAM; ROESLI, 2000). Segundo esses autores, a morte dos insetos pela terra de diatomácea é atribuída à dessecação provocada pela adsorção e abrasividade deste pó inerte que rompe a camada de cera da epicutícula dos insetos, fazendo com que eles percam água do corpo até morrerem.

0 uso de protetores de grãos na região Norte do Brasil é raro. Nesse sentido, o objetivo deste trabalho foi avaliar a eficiência de terra de diatomácea, aplicada em milho armazenado no Estado de Roraima, no controle de T. castaneum.

\section{MATERIAISE MÉTODOS}

Grãos do híbrido de milho BRS 2020 foram tratados com seis dosagens de terra de diatomácea (860 g. $\mathrm{kg}^{1}$ de dióxido de sílica), 0, 200, 400, 600, 800 e 1000 g.t $^{-1}$, com quatro repetições. Cada tratamento, contendo $100 \mathrm{~g}$ de grãos, mantido em pote de vidro telado, foi infestado com 30 
adultos de T. castaneum e mantido em condicões de laboratório a $27^{\circ} \mathrm{C}$. A mortalidade acumulada foi avaliada do primeiro até o vigésimo oitavo dia após a infestação.

O modelo adotado para analisar os dados considerou o efeito da dosagem e o tempo de exposição da terra de diatomácea sobre a mortalidade dos insetos. O s dados de mortalidade foram submetidos à análise de variância e as médias comparadas por meio do teste de Duncan em $5 \%$. O s dados foram analisados utilizando-se o SAS ${ }^{\circledR}$ System e o STATISTICA 5.5 (LITTEL et al., 1996, STATSOFT INC., 1999).

O comportamento de mortalidade, ao longo do tempo, foi desdobrado em cada um dos níveis marcados como significante no modelo, sob a aplicação de um ajuste não linear do tipo logístico $\mathrm{y}=\mathrm{a} /\left(1+\left(\mathrm{x} / \mathrm{x}_{0}\right)^{\mathrm{b}}\right)$. 0 co eficiente de determinação ajustado $\left(\mathrm{R}_{\text {aj. }}^{2}\right)$ foi usado como indicador de aderência do modelo em relação às observações.

\section{RESULTADOS}

O bservou-se, neste trabalho, uma tendência de aumento da mortalidade de T. castaneum à medida que se aumentou o tempo de exposição dos insetos à terra de diatomácea (Figura 1 e Tabela 1). Resultados semelhantes também foram encontrados por D owdy (1999) e Arthur (2001). O s autores observaram aumento na mortalidade de T. castaneum e 0 . surinamensis quando aumentaram o período de exposição dessas pragas ao pó inerte.

0 aumento da mortalidade dos insetos com o aumento das dosagens, observada neste trabalho (Figura 1 e Tabela 1), também foi verificado por Athanassiou et al. (2005a). Os autores trataram trigo com terra de diatomácea em dosagens crescentes, de 0 até 1500 g.t $^{-1}$, e constataram que houve um incremento na mortalidade de T. confusum à medida que se aumentou a dosagem do pó inerte.

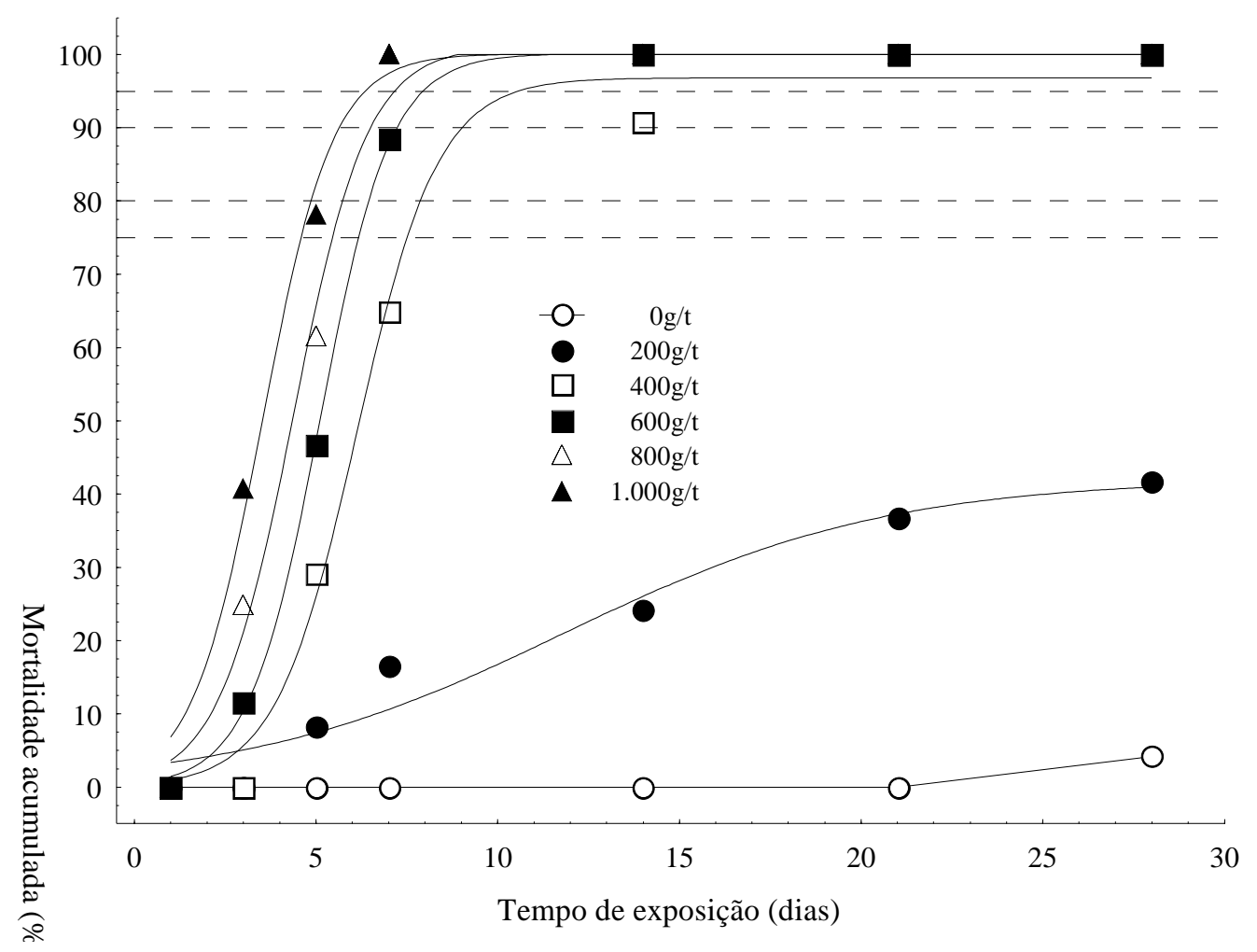

FIGURA 1 - Ajuste do modelo logístico à mortalidade acumulada em função dos dias de exposição dos insetos à terra de diatomácea 
MARSARO Jr., A. L. et al.

TABELA 1 - Valores médios (\%)* da mortalidade acumulada de Tribolium castaneum em função do tempo de exposição dos insetos à terra de diatomácea e suas dosagens, bem como os coeficientes do modelo logístico ajustado

\begin{tabular}{|c|c|c|c|c|c|c|c|c|c|c|c|}
\hline \multicolumn{12}{|c|}{ D osagens da terra de diatomácea (g/t) } \\
\hline Dias & $\mathbf{0}$ & & 200 & & 400 & & 600 & & 800 & 1000 & \\
\hline 3 & 0,0 & d & 0,0 & d & 0,0 & $d$ & 11,7 & c & $25,0 \quad b$ & 40,8 & $\mathrm{a}$ \\
\hline 5 & 0,0 & $\mathrm{f}$ & 8,3 & e & 29,2 & d & 46,7 & C & $61,7 \quad b$ & 78,3 & a \\
\hline 7 & 0,0 & $\mathrm{e}$ & 16,7 & d & 65,0 & C & 88,3 & $\mathrm{~b}$ & $100,0 \mathrm{a}$ & 100,0 & $\mathrm{a}$ \\
\hline 14 & 0,0 & $\mathrm{~d}$ & 24,2 & $\mathrm{C}$ & 90,8 & $\mathrm{~b}$ & 100,0 & $\mathrm{a}$ & $100,0 \mathrm{a}$ & 100,0 & $\mathrm{a}$ \\
\hline 21 & 0,0 & C & 36,7 & $\mathrm{~b}$ & 100,0 & a & 100,0 & a & $100,0 \mathrm{a}$ & 100,0 & a \\
\hline 28 & 4,2 & c & 41,9 & $\mathrm{~b}$ & 100,0 & $\mathrm{a}$ & 100,0 & $\mathrm{a}$ & $100,0 \mathrm{a}$ & 100,0 & a \\
\hline \multicolumn{12}{|c|}{ Coeficientes e parâmetros do modelo } \\
\hline & $\mathbf{0}$ & & 200 & & 400 & & 600 & & 800 & 1000 & \\
\hline $\mathrm{a}$ & $\sim$ & & 0,420 & & 0,968 & & 1,001 & & 1,012 & 1,001 & \\
\hline b & $\sim$ & & 4,452 & & 1,121 & & 0,975 & & 1,024 & 0,967 & \\
\hline $\mathrm{x}_{0}$ & $\sim$ & & 11,838 & & 6,124 & & 5,105 & & 4,369 & 3,525 & \\
\hline $\mathrm{R}^{2}$ & $\sim$ & & 0,95 & & 0,99 & & 0,99 & & 0,99 & 0,99 & \\
\hline $\mathrm{R}_{\text {aj. }}^{2}$ & $\sim$ & & 0,93 & & 0,99 & & 0,99 & & 0,99 & 0,99 & \\
\hline
\end{tabular}

*Valores seguidos pela mesma letra, na horizontal, não diferem estatisticamente entre si, pelo teste de Duncan $\left(\mathrm{pd}^{\prime \prime} 0,05\right)$.

A mortalidade ocorreu mais rapidamente nas dosagens mais elevadas, 800 e 1000 g.t $^{-1}$ (Tabela 1 e Figura 1). Na menor dosagem, 200 g.t ${ }^{1}$, mesmo após 28 dias de exposição, não houve um controle total da população de insetos. D osagens baixas de terra de diatomácea necessitam de um período maior de exposição dos insetos aos pós inertes para causar a mortalidade e isso pode favorecer o desenvolvimento de uma segunda geração de insetos, conforme já observado por Marsaro Jr. et al. (2006).

0 modelo logístico usado (Tabela 1) estimou o tempo para obter $95 \%$ de mortalidade da população de insetos, para a dosagem de 1000 g.t $^{-1}$, de 6 dias, para a dosagem de 800 g.t-1 , de 7 dias, enquanto nas dosagens de 600 e 400 g.t $t^{-1}$ foram necessários 8 e 10 dias, respectivamente. Resultados semelhantes foram encontrados por A thanassiou et al. (2005a). Utilizando a dosagem de $1000 \mathrm{~g}^{-1}{ }^{-1}$ desse pó inerte os autores verificaram mortalidade acima de $95 \%$ da população de T. confusum após 7 dias de exposição dos insetos à terra de diatomácea.

$\mathrm{Na}$ menor dosagem utilizada de terra de diatomácea, 200 g.t $\mathrm{t}^{-1}$, a mortalidade máxima alcançada não ultrapassou 42\%, mesmo após 28 dias de exposição dos insetos ao pó inerte. Usando essa mesma dosagem, Arnaud et al. (2005) constataram mortalidade inferior a 10\% da população de T. castaneum, mesmo após 21 dias de exposição dos insetos ao protetor de grãos.

Mewis e Ulrichs (2001) e A thanassiou et al. (2005a) também verificaram que, em baixas dosagens, para se alcançar um satisfatório nível de controle de pragas de grãos armazenados, um maior perío do de exposição dos insetos aos pós inertes é necessário. Esse fato está relacionado com o modo de ação da terra de diatomácea sobre o inseto. De acordo com Subramanyam e Roesli (2000) a morte dos insetos pela terra de diatomácea é atribuída à dessecação provocada pela adsorção e abrasividade deste pó inerte que rompe a camada de cera da epicutícula dos insetos, fazendo com que eles percam água do corpo até morrerem. Portanto, em altas dosagens, a adsorção e a abrasividade causadas pela terra de diatomácea ocorrem mais rapidamente, causando a morte num curto intervalo de tempo, quando comparado com as menores dosagens. 
Produtos compostos por terra de diatomácea usualmente necessitam de um tempo um pouco maior para matar os insetos, quando comparado com os inseticidas que agem por contato, entretanto, 0 efeito residual da terra de diatomácea é usualmente maior. Estudos realizados por A thanassiou et al. (2005b) demonstraram que a terra de diatomácea causou mais de 90\% de mortalidade da população de S. oryzae em trigo tratado com esse protetor por um período superior a 270 dias.

\section{CONCLUSÕES}

A terra de diatomácea apresentou uma alta eficiência no controle de T. castaneum, nas dosagens de 800 e 1000 g.t ${ }^{-1}$, num curto espaço de tempo.

D osagens mais baixas de terra de diatomácea necessitam de maior período de exposição dos insetos ao pó inerte para alcançarem níveis de mortalidade significativos.

\section{REFERÊNCIAS}

ALD RY HIM, Y. N. Efficacy of the amorphous silica dust, dryacide, against Tribolium confusum Duv. and Sitophilus granarius (L.) (Coleoptera: Tenebrionidae and Curculionidae). J. Stored Prod. Res., London, v. 26, n. 4, p. 207-210, 1990.

ARNAUD, L. et al. Efficacy of diatomaceous earth formulations admixed with grain against of Tribolium castaneum. J. Stored Prod. Res., London, v. 41, n. 2, p. 121-130, 2005.

ARTHUR, F. H. Immediate and delayed mortality of 0 ryzaephilus surinamensis (L.) exposed on wheat treated with diatomaceous earth: effects of temperature, relative humidity, and exposure interval. J. Stored Prod. Res., London, v. 37, n. 1, p. 13-21, 2001.

ATHANASSIOU, C. G. et al. Insecticidal efficacy of diatomaceous earth against Sitophilus oryzae (L.) (Coleoptera: Curculionidae) and Tribolium confusum du Val (Coleoptera: Tenebrionidae) on stored wheat: influence of dose rate, temperature and exposure interval. J. Stored Prod. Res., London, v. 41, n. 1, p. 47-55, 2005a.

. Persistence and efficacy of three diatomaceous earth formulation against Sitophilus oryzae (C̄oleoptera: Curculionidae) on wheat and barley. J. Econ. Entomol., California, v. 98, n. 4, p. 1404-1412, 2005b.

BALDASSARI, N.; BALDONI, G.; BARO NIO, P. Efficacy of different diatomaceous earths to control adult insects. Tec. Molit., Italy, v. 53, p. 1201-1207, 2002.

BELLO, G. et al. Biocontrol of A canthoscelides obtectus and Sitophilus oryzae with diatomaceous earth and Beauveria bassiana on stored grains. Biocontrol Sci. Technol., London, v. 16, n. 1, p. 215-220, 2006.

COLLINS, D. A.; COOK, D. A. Laboratory evaluation of diatomaceous earths, when applied as dry dust and slurries to wooden surfaces, against stored-product insect and mite pests. J. Stored Prod. Res., London, v. 42, n. 2, p. 197-206, 2006.

DOWDY, A. K. Mortality of red flour beetle, Tribolium castaneum (Coleoptera: Tenebrionidae) exposed to high temperature and diatomaceous earth combinations. J. Stored Prod. Res., London, v. 35, n. 2, p. 175-182, 1999.

LITTEL, R. C. et al. SA S ${ }^{\circledR}$ System for Mixed Models. SA S Institute Inc., Cary, 1996.

LORINI, I. Manual técnico para o manejo integrado de pragas de grãos de cereais armazenados. Passo Fundo: Embrapa Trigo, 2001. 
MARSARO Jr., A. L. et al. Interação entre híbridos de milho e protetores de grãos no controle de Sitophilus zeamais Motschulsky (Coleoptera: Curculionidae). Rev. Bras. Armazen., Viçosa, v. 31, n. 2, p. 144-153, 2006.

MEWIS, I.; ULRICHS, C. Action of amorphous diatomaceous earth against different stages of the stored product pests Tribolium confusum, T enebrio molitor, Sitophilus granarius and Plodia interpunctella. J. Stored Prod. Res., London, v. 37, n. 2, p. 153-164, 2001.

STATHERS, T. E.; DENNIFF, M.; GOLOB, P. The efficacy and persistence of diatomaceous earth admixed with commodity against four tropical stored product beetle pests. J. Stored Prod. Res., London, v. 40, n. 2, p. 113-123, 2004.

STATSO FT, INC. Statistica for Windows [Computer program manual]. Tulsa: StatSoft, Inc., 1999. D isponível em: <http:// www.statsoft.com>. Acesso em: 26 ago. 2008.

SUBRAMANYAM, B.; ROELSI, R. Inert dusts. In: SUBRAMANYAM, B.; HAGSTRUM, D. W. (Ed.). Alternatives to pesticides in stored-product IPM. Norwell: Kluwer Academic Publishers, 2000. p. 321-380.

Recebido: $15 / 03 / 2008$

Received: $03 / 15 / 2008$

Aprovado: 10/ 11/ 2008

A pproved: $11 / 10 / 2008$

Revisado: 19/ 08/ 2009

Reviewed: 08/ 19/2009 